\title{
Dynamic Analysis of Draft Gear and Draft Pad of Freight Wagon due to Localized Defects using FEM
}

\author{
Sachin S. Harak, Satish C. Sharma and S. P. Harsha \\ Vibration and Noise Control Lab., Mechanical \& Industrial Engineering Department, Indian Institute of Technol- \\ ogy, Roorkee - 247667, Uttarakhand State, India
}

(Received 26 November 2013; accepted 12 October 2015)

\begin{abstract}
The present work investigates the effect of a crack on the modal frequency of a draft pad. Initially, the first five mode shapes of a healthy draft pad and the first seven mode shapes of healthy draft gear considering compressed draft pads are determined using the finite element approach. A mathematical model of the draft pad is formulated to predict the effect of the crack on its modal frequency. A semi-elliptical shaped crack is modelled in the lateral and longitudinal direction of the draft pad. It is observed that if the crack lies in the zone of minimum modal displacement, then the frequency drop is minimal, and if the crack lies in the zone of maximum modal displacement, then the frequency drop is significant. Various damage scenarios are simulated by varying the width and aspect ratio of the crack in order to identify its effect on the modal frequency. It is seen that if the aspect ratio is varied while the crack's width is maintained constant, then the frequency drop is linear, whereas if the crack's width is varied while the aspect ratio is maintained constant, then the frequency drop is parabolic. This study provides a tool for monitoring exciting frequencies of draft gear and shows how each modal frequency is affected by the crack due to parameters like aspect ratio, crack width, and crack location/orientation.
\end{abstract}

\section{INTRODUCTION}

The longitudinal dynamics of freight wagons largely depends on the dynamics of draft gear and consequently the draft $\operatorname{pad}(\mathrm{s})$. Draft gear is a key part of autocouplers in freight wagons, as they function like a cushioning device absorbing shocks in the form longitudinal forces arising due to train operations like accelerating or braking. The draft gear (RF-361) under consideration is manufactured by Miner Enterprises Inc., USA. It is normally used in open hopper/coal wagons and bulk commodity wagons. Freight wagons in Indian Railways are loaded with RF-361 draft gear. The various components of the draft gear illustrated in Fig. 1a are six draft pads with a top follower, three shoes, and a wedge. The draft pad shown in Fig. 1b consists of a rubber compound sandwiched between two steel plates and is perfectly bonded to them. All these components are assembled in housing with the three shoes arranged circumferentially around the wedge. The wedge and shoes operate between the top follower and the draft gear housing. The applied forces reach the draft pads through the wedge and shoes. Each pad has an ultra-high capacity natural rubber spring package to absorb the high longitudinal forces arising due to operations like acceleration and/or braking and also due to changes in track topography. These forces, being repetitive in nature, accelerate the damage to the draft pads in the presence of defects. These draft pads, when used in damaged conditions, cause the longitudinal forces to be transmitted to the wagon, which compromises the safety of the laden goods.

A review shows that the longitudinal in-train forces are responsible for several problems including broken draft gear and even causing freight wagons to be pulled off the inside of curves. ${ }^{1}$ Initial efforts by researchers were aimed at reducing longitudinal oscillations in passenger trains. Measurements and simulations of in-train forces were carried out by Duncan and Webb to achieve reduction in longitudinal oscillations. ${ }^{2}$ Work by Chen was aimed at developing a mathematical model to calculate transient responses of a coupler and to identify

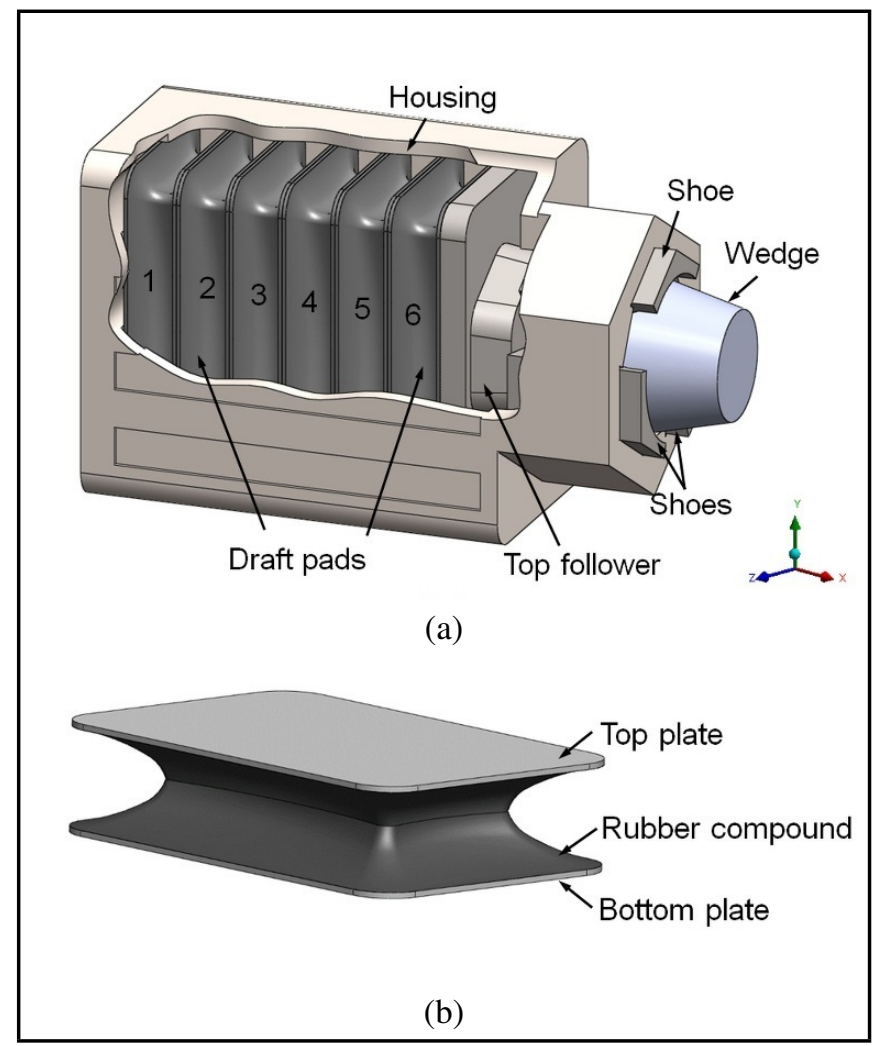

Figure 1. (a) A cross-sectional view of draft gear unit (RF-361) and (b) a typical draft pad (RF-8).

the coupling speed. ${ }^{3}$ Experimental efforts by McClanachan et al. helped determine the occurrences of coupler impacts combined with pitching motions in the wagon body. ${ }^{4}$ The impacts were simulated using the train-wagon interaction model in NUCARS and ADAMS/Rail. The fatigue life of three different wagon connection coupling systems that had draft gear with and without self-locking features was evaluated by Cole 
and Sun. ${ }^{5}$ A numerical procedure was developed by Belforte et al. to investigate the dynamics of heavy freight trains. ${ }^{6}$ The proposed methodology by Belforte et al. combined a numerical model designed for the longitudinal dynamics of the whole trainset called TrainSet Dynamics simulator (TSDyn) with a multibody code named Modular Non-Stationary TRAMcar dynamics simulator originally developed to analyse the dynamics of modular tramcars (MoNSTram) and used for a more detailed investigation of the response of a trainset segment.

The effect of train braking delay time (for long, medium, and short brake application time) at different train forward velocities on longitudinal dynamics of trains was studied by Nasr and Mohammadi. ${ }^{7}$ Cole et al. added modelling of coupler angles to a normal longitudinal simulation for a comprehensive study of lateral components of coupler forces, as these forces affected the stability of long and heavier trains. ${ }^{8}$ Qi et al. improved the efficiency and safety of the wagons during positioning operations by optimizing the design speed of the positioner based on the simulation of longitudinal train dynamics. This was achieved by replacing the traditional draft gear model with nonlinear spring and linear damping with a more detailed model based on the achievement of contact and impact mechanics while considering the coupler slack. ${ }^{9}$ Recently, Wu et al. developed a new technology called the Train dynamics and energy analyser/train simulator (TDEAS) by modelling three aspects: wagon connection systems, air brake systems, and train energy components in order to perform detailed whole trip longitudinal train dynamics and energy analyses and to act as a driving simulator for heavy haul trains. ${ }^{10}$ The simulator uses advanced wedge-spring draft gear models that can simulate a wider spectrum of friction draft gear behaviour.

The present work concentrates on the effect of a crack on the natural frequency of a draft pad, as it plays a significant role in predicting the fatigue behaviour of the components subjected to repetitive loads. Initially the first five mode shapes of a healthy draft pad and the first seven mode shapes of healthy draft gear were determined by using the finite element approach. These mode shapes were compared to establish the participating mode shapes of the draft pad in the draft gear. Furthermore, the effect of the crack on participating mode shapes of the draft pad was determined by using the finite element approach. A mathematical model of the draft pad is formulated to predict the effect of the crack on its modal frequency. The crack was modelled by using a semi-elliptical shape in two directions, viz., lateral and longitudinal direction of the draft pad. Various damage scenarios were simulated by varying the width as well as the aspect ratio of the crack to identify its effect on modal frequency.

\section{MODAL ANALYSIS USING FINITE ELEMENT APPROACH}

Modal analysis determines the natural frequencies and mode shapes of a continuous structure. For a free vibration analysis, the natural frequencies $\left(\omega_{i}\right)$ and the mode shapes $\left(\phi_{i}\right)$ were calculated using the following relation: $\left([K]-\omega_{i}^{2}[M]\right) \phi_{i}=0 .{ }^{11}$ Here, the finite element was used to setup the system equations of motion, which were solved for natural frequencies and mode shapes using Eigen-value analysis. For evaluating mode shapes, the material was assumed to behave linearly elastic neglecting nonlinearities.

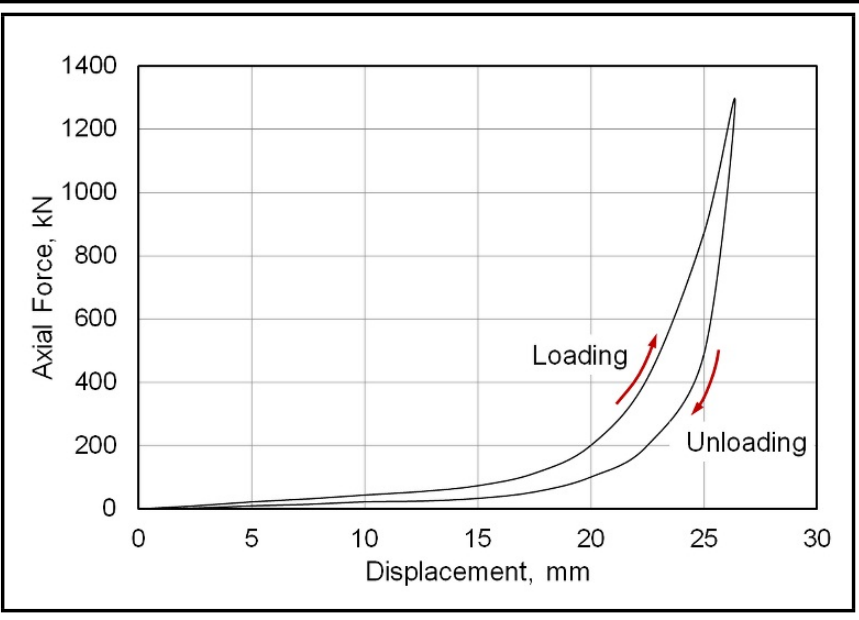

Figure 2. A typical manufacturer's draft pad response data.

\subsection{Model For Modal Analysis}

For vibration-based analysis, which is model dependent, an accurate numerical model was important to use. Thus, three dimensional models of the draft pad and draft gear were created using Solidworks and exported to ANSYS environment for numerical analysis.

\subsubsection{Draft pad}

The draft pad used in this study is illustrated in Fig. 1b. It is available commercially as "RF-8" and confirms to "Drg. No. WD-90076" of Indian Railways. The draft pad consists of a rubber compound sandwiched between two steel plates and is perfectly bonded to them. The material properties of steel are Young's modulus: $200 \times 10^{9} \mathrm{~N} / \mathrm{m}^{2}$, Poisson's ratio: 0.30 , and material density: $7850 \mathrm{~kg} / \mathrm{m}^{3}$. The material properties for the rubber compound were derived from experimental data, which was a non-linear plot of force and displacement as illustrated in Fig. 2. This plot is an outcome of a uniaxial test on a draft pad. Young's modulus and Poisson's ratio were determined with the help of a material model calibration software. The hysteresis due to visco-elastic behaviour of rubber compound was responsible for dissipating the longitudinal shock loads. The damping ratio $(\zeta)$ was evaluated to be 0.05 and the same was considered during simulation. The numerical values are: Young's modulus $=21.94 \times 106 \mathrm{~N} / \mathrm{m}^{2}$ and Poisson's ratio $=$ 0.4978 . The material density was specified by the manufacturer and is $1220 \mathrm{~kg} / \mathrm{m}^{3}$. The effective life span of natural rubber under operating conditions was considered to be 15 years. However, periodic maintenance was scheduled every five years and includes replacement of failed draft pads. ${ }^{12}$ This indicates that the average life of almost all draft pads is around five years. Hence, changes in the properties of rubber compounds due to ageing were not included for the proposed dynamic analysis. The boundary condition for modal analysis of a draft pad thus included fixing all nodes at the outer surface of bottom plate.

\subsubsection{Draft gear}

Fig. 1a illustrates the sectional view of draft gear. The various components of the draft gear consisted of six draft pads with a top follower, three shoes, and a wedge. The material properties taken were the same as those in the earlier case. For the boundary conditions, all nodes at the bottom surface of the housing were fixed, whereas the interface between adjacent pads were assumed to be bonded type since the pads were 


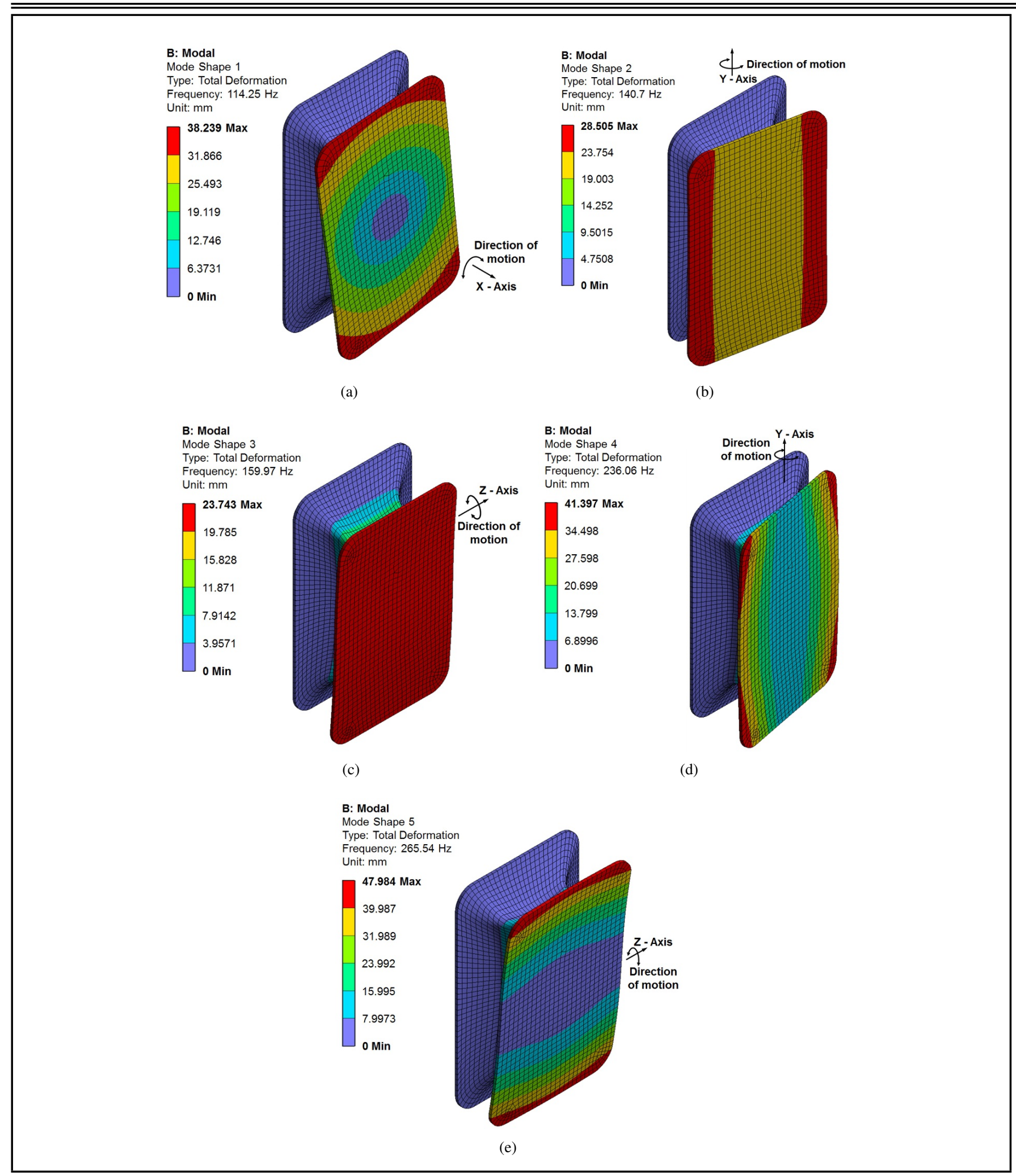

Figure 3. Mode shapes of the draft pad: (a) the first mode shape at $114.25 \mathrm{~Hz}$ (b) the second mode shape at $140.70 \mathrm{~Hz}$ (c) the third mode shape at $159.97 \mathrm{~Hz}$ (d) the fourth mode shape at $236.06 \mathrm{~Hz}$ and (e) the fifth mode shape at $265.54 \mathrm{~Hz}$.

in constant compression within the draft gear.

\subsection{Mode Shapes and Frequencies of Draft Pad and Draft Gear}

The modal frequencies of an individual draft pad (for the rst five modes) and draft gear (for first seven modes) were obtained using the finite element package (ANSYS) and presented in Tables 1 and 2 respectively.
Table 1. The first five modal frequencies of an individual draft pad in $\mathrm{Hz}$.

\begin{tabular}{|c|c|c|c|c|c|}
\hline Mode & 1 & 2 & 3 & 4 & 5 \\
\hline Frequency & 114.25 & 140.7 & 159.97 & 236.06 & 265.54 \\
\hline
\end{tabular}

Table 2. The first seven modal frequencies of draft gear in $\mathrm{Hz}$.

\begin{tabular}{|c|c|c|c|c|c|c|c|}
\hline Mode & 1 & 2 & 3 & 4 & 5 & 6 & 7 \\
\hline Frequency & 61.97 & 69.99 & 77.75 & 121.12 & 132.88 & 147.97 & 174.22 \\
\hline
\end{tabular}


The first five mode shapes of an individual draft pad are illustrated in Fig. 4. Hence, it is seen that:

- The first mode shape, Fig. 3a, describes oscillatory motion of the rubber compound and the top plate about the $\mathrm{x}$-axis of the draft pad.

- The second mode shape, Fig. 3b, shows oscillation of the rubber compound and the top plate about the yaxis. However, the $y$-axis about which the oscillation occurs doesnt coincide with the draft pad $y$-axis but passes through the base plate of the draft pad.

- The third mode shape, Fig. 3c, describes oscillation of the rubber compound and the top plate about the z-axis. The z-axis doesn't coincide with the draft pad z-axis but passes through the base plate of draft pad.

- The fourth mode shape, Fig. 3d, shows oscillation of the rubber compound and the top plate about the y-axis. The $y$-axis is contained by the top plate, as seen in the illustration.

- The fifth mode shape, Fig. 3e, indicates oscillatory motion of the rubber compound and the top plate z-axis. As seen in the illustration, the $\mathrm{z}$-axis is contained by the top plate.

A comparison of the mode shapes of each draft pad in the draft gear with mode shapes of an individual draft pad was done. It was observed that the individual draft pad's rst ve mode shapes dominated the behaviour of each draft pad in the draft gear for all seven mode shapes (of the draft gear). This was because the draft pads housed in the draft gear had their own individual behaviour, which cumulatively resulted in different mode shapes of the draft gear. These mode shapes of the draft gear had no resemblance with mode shapes of an individual draft pad. Fig. 4a describes the rst mode shape of a draft gear. The draft gear housing was very stiff compared to the draft pad, and thus had a very poor modal response. Hence, it had been hidden in this view. The modal behaviour of each draft pad was presented separately. It was seen that all the draft pads oscillated about the longitudinal (x) axis of the draft gear. Each draft pad exhibited modal behaviour close to its rst mode shape. A symmetry in modal behaviour was observed at the interface of the third and fourth pads for all seven mode shapes of the draft gear. Hence, the modal behaviour of only the rst three draft pads is presented in the illustrations.

For the second mode of the draft gear, a linear displacement of the interface between pad 3 and pad 4 in the lateral (z) direction was observed, which caused the draft pads to exhibit modal behaviour close to its second mode shape. The third mode showed linear displacement of the interface between pad 3 and pad 4 in the vertical (y) direction, causing the draft pads to exhibit modal behaviour close to its third mode shape. For the fourth mode shape, the interface between pad 3 and pad 4 remained fixed, and the interfaces between the pads ( 1 and 2; pads 2 and 3; pads 4 and 5; and pads 5 and 6) oscillates about the longitudinal (x) axis in the opposite sense. As a result, modal behaviour corresponding to the first mode shape of the draft pad was observed in some of the draft pads.

The fifth mode shape showed oscillation of the interface between pad 3 and pad 4 about the vertical (y) axis. The result was that some draft pads exhibited fourth modal behaviour of an individual draft pad. The remaining draft pads exhibited higher modal behaviour. The sixth mode shape showed oscillation of the interface between pad 3 and pad 4 about the lateral (z) axis. As a result, some draft pads exhibited third modal behavior while some exhibited fifth modal behaviour of an individual draft pad. In the seventh mode shape, the interfaces between pads 2 and 3 and pads 4 and 5 remained fixed, and the interfaces between pads 1 and 2; 3 and 4; and 5 and 6 oscillated about the longitudinal ( $\mathrm{x}$ ) axis, which caused every single pad to execute motion in accordance to the first mode shape. The oscillatory motion of the interface between pads 1 and 2, 5 and 6, and 3 and 4 was opposed. This modal relationship between pads in the draft gear as well as an individual draft pad is summarized in Table 3.

\section{THE EFFECT OF CRACKS ON MODAL FREQUENCIES}

As per the fundamentals of vibration, the natural frequency $\left(\omega_{n}\right)$ for any mechanical system is affected by two parameters: mass $(m)$ and stiffness $(K)$. The effect of these two parameters is generally defined by the equation:

$$
\omega_{n}=\sqrt{K / m} .
$$

The natural frequency is directly proportional to the stiffness of the system. Thus, any parameters that affect the stiffness of the system also affect its natural frequency. Hooke's law can also be used to correlate system stiffness with other parameters. As per Hooke's law:

$$
\sigma=E \cdot \varepsilon
$$

or

$$
\frac{W}{A}=E \frac{\delta l}{l} .
$$

The terms in Eqs. (2) and (3) carry their usual meaning. The stiffness for any system that obeys Hookes law can thus be written as:

$$
K=\frac{W}{\delta l}=E \frac{A}{l} .
$$

It is seen that the stiffness of any system is affected by its Young's modulus, cross-sectional area, and thickness. Thus, these parameters have a similar impact on the system's natural frequency. The crack's presence affects the cross-sectional area of the system and tends to reduce it. A reduced area results in reduced stiffness and, consequently, a reduced natural frequency, which is seen in Eqs. (1) and (4).

While considering any crack, variation in the area of a crack is effected by the crack's width and aspect ratio. When the crack's width is constantly maintained and the aspect ratio is varied, it is seen that the area is the function of a single parameter:

$$
A=f \text { (depth of crack). }
$$

From Eq. (5) it is observed that changing the aspect ratio while maintaining the crack width constant results in linear variation of the area. If the variation in area is effected by varying the crack width and maintaining aspect ratio constant, then area of the crack is function of two parameters, viz., crack width and depth of crack. Thus,

$$
A=f(\text { crack width, depth of crack }) .
$$




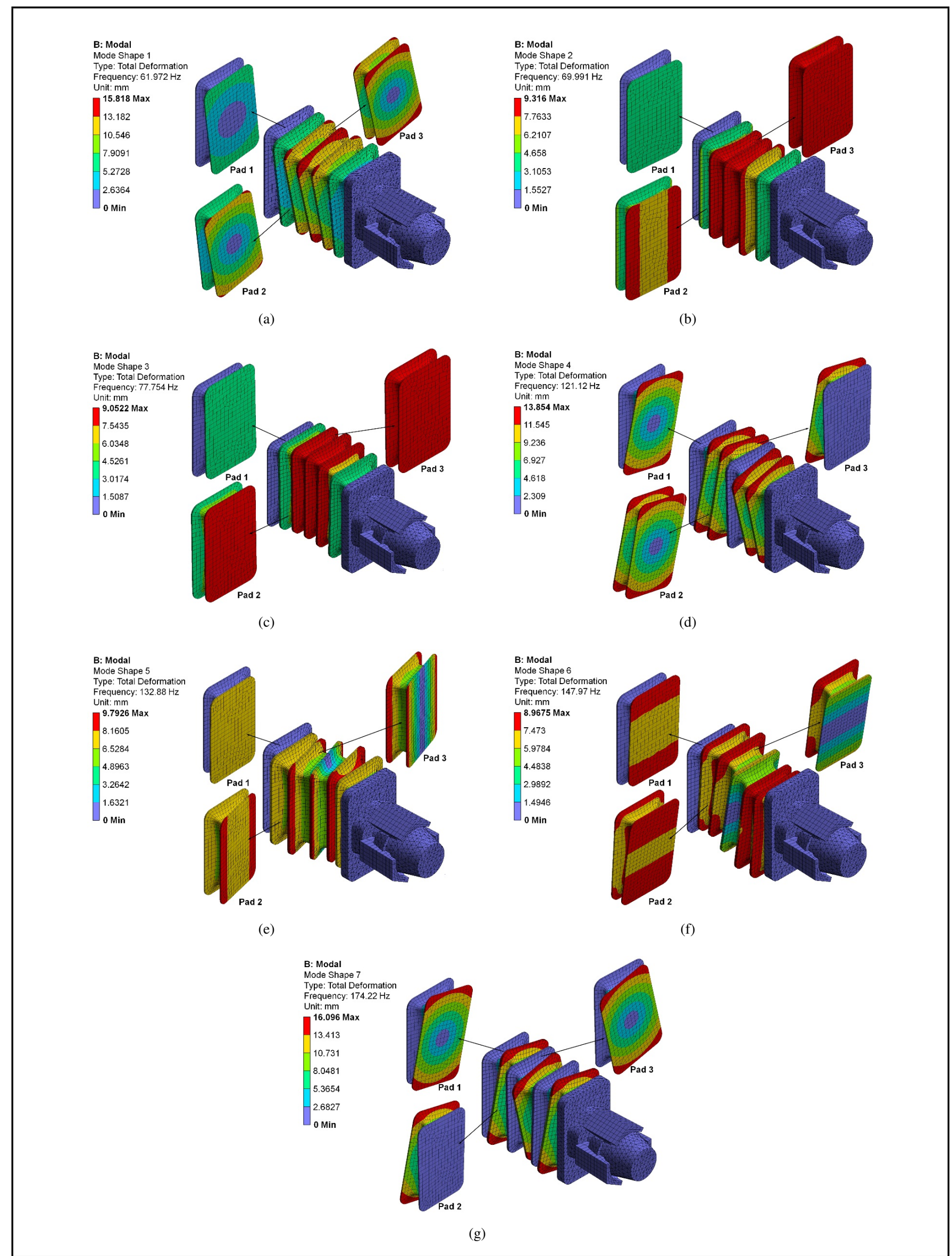

Figure 4. Mode shapes of the draft gear with housing hidden for the (a) first modal frequency (b) the second modal frequency (c) the third modal frequency (d) the fourth modal frequency (e) the fifth modal frequency (f) the sixth modal frequency and (g) the seventh modal frequency. 
Table 3. Participating mode shapes of an individual draft pad in the modal behaviour of draft gear.

\begin{tabular}{|c|c|c|c|c|c|c|c||}
\hline Draft gear & & \multicolumn{6}{|c|}{ Participating modes of various draft pads (of draft gear) } \\
\hline Mode No. & Frequency & Pad 1 & Pad 2 & Pad 3 & Pad 4 & Pad 5 & Pad 6 \\
\hline 1 & 061.97 & Mode 1 & Mode 1 & Mode 1 & Mode 1 & Mode 1 & Mode 1 \\
\hline 2 & 069.99 & Mode 2 & $*$ & Mode 2 & Mode 2 & $*$ & Mode 2 \\
\hline 3 & 077.75 & Mode 3 & Mode 3 & $*$ & $*$ & Mode 3 & Mode 3 \\
\hline 4 & 121.12 & Mode 1 & $*$ & Mode 1 & Mode 1 & $*$ & Mode 1 \\
\hline 5 & 132.88 & $*$ & Mode 4 & $*$ & $*$ & Mode 4 & $*$ \\
\hline 6 & 147.97 & $*$ & Mode 5 & Mode 3 & Mode 3 & Mode 5 & $*$ \\
\hline 7 & 174.22 & Mode 1 & Mode 1 & Mode 1 & Mode 1 & Mode 1 & Mode 1 \\
\hline
\end{tabular}

Under such circumstances, variation in the area is either parabolic or second order. This reduction in stiffness will consequently reduce the natural frequency. Thus, for a draft pad, the presence of a crack should cause a reduction in natural frequency, as seen in Eqs. (5) and (6).

\subsection{Mathematical Model of Draft Pad}

As discussed earlier, the effect of the crack was to reduce the stiffness of the system. Hence, a mathematical relationship between the natural frequency and the stiffness of the system was necessary to predict the idealized behaviour of the system in the presence of the crack. From simulation, it was observed that all the mode shapes of the draft pad exhibited an oscillatory motion about either the $\mathrm{x}, \mathrm{y}$, or $\mathrm{z}$ axis. The same could also be observed in Fig. 4. All of these modal behaviours could be approximated by a single degree of freedom model as shown in Fig. 5a. The bottom plate was grounded and the mass of the top plate and the rubber compound was lumped as a single mass and represented as $m_{p}$. The stiffness of the rubber compound was represented by two stiffnesses, $K_{1}$ and $K_{2}$, such that:

$$
K_{\text {pad }}=K_{1}+K_{2}
$$

The mass was free to oscillate about its centroid. The stiffnesses, $K_{1}$ and $K_{2}$, are located at distances $a$ and $b$ respectively from the centroid and represent the crack's location in the plane of the crack. This proposed model was used to determine the parameters affecting the modal frequency.

Figure $5 \mathrm{~b}$ shows the forces acting on the draft pad. Here, $K_{1} . a \theta$ and $K_{2} . b \theta$ are the restoring forces opposing the inertia couple $I \ddot{\theta}$. The equation of motion for the mass $m_{p}$ can be determined by using D'Alembert's principle:

$$
I \ddot{\theta}=-K_{1} \cdot a \theta \cdot a-K_{2} \cdot b \theta \cdot b ;
$$

or

$$
I \ddot{\theta}+\left(K_{1} \cdot a^{2}+K_{2} \cdot b^{2}\right) \theta=0 ;
$$

and

$$
\omega_{n}=\sqrt{\frac{\left(K_{1} \cdot a^{2}+K_{2} \cdot b^{2}\right)}{I}} .
$$

It can be seen from Eq. (10) that the stiffnesses, $K_{1}$ and $K_{2}$, of the mass $m_{p}$ directly impacted the natural frequency of the system. Thus, the presence of the crack that reduced the area caused a reduction in stiffness and consequently the natural frequency. Hence, for the draft pad, the natural frequency was dependent on the presence of the crack (in the form of reduced values of stiffnesses $K_{1}$ or $K_{2}$ ) and the location of the crack in its own plane.

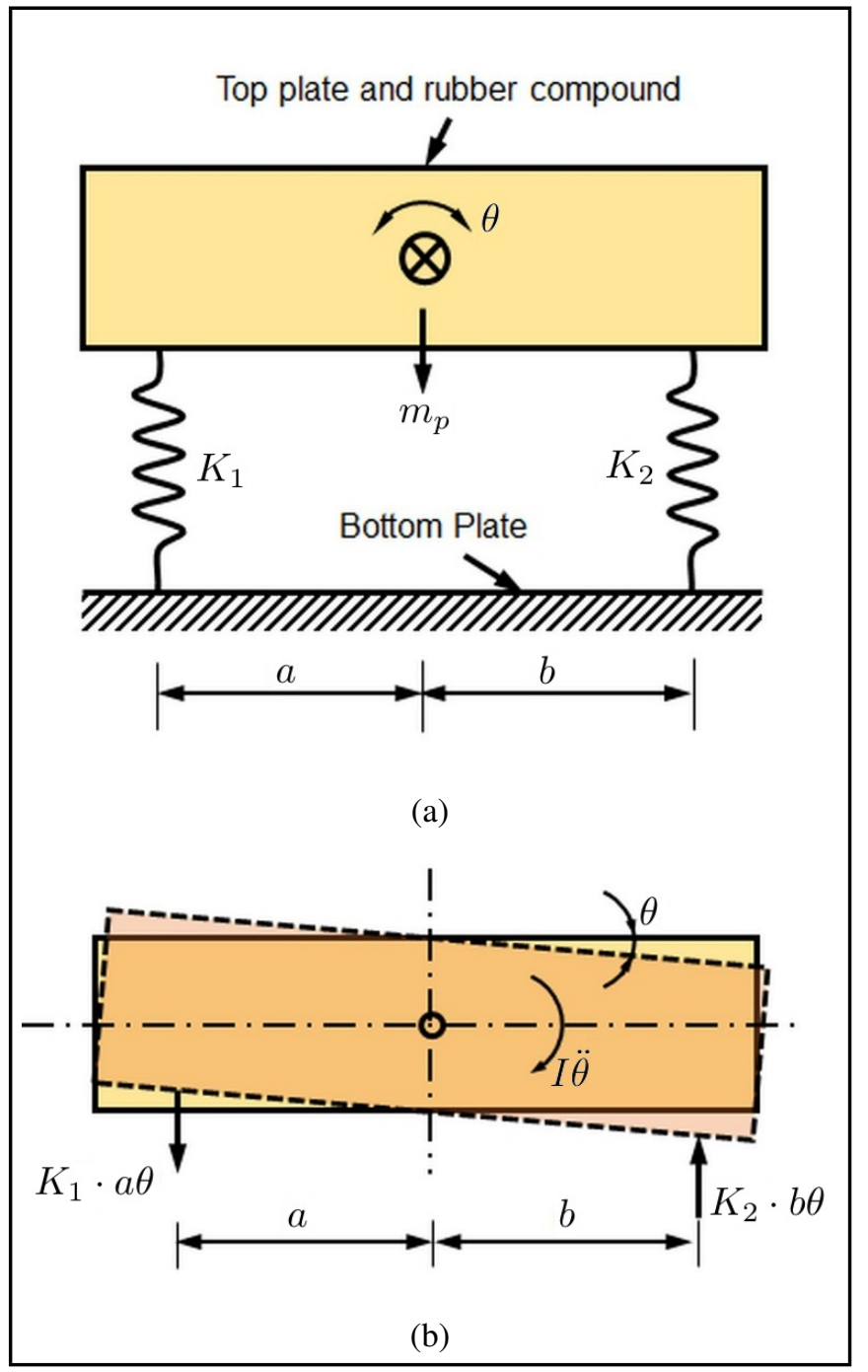

Figure 5. (a) The mathematical model of the draft pad and (b) the free body diagram of the draft pad.

\subsection{Modelling a Crack in 3D CAD Model of Draft Pad}

An analysis of the types of pad failures was carried out in a maintenance workshop of Indian Railways located in Jagadhri, Haryana State, India, which is the largest amongst those in the northern part of the country. It handles as much as 40 draft gear units daily, apart from other maintenance activities. The shape of the crack was finalized after a careful analysis of the draft pads, which failed during operation. The following are some important illustrations that were instrumental in finalizing the shape and location of the cracks.

As reported by the operators in the workshop, nearly $80 \%$ of the draft pads that were replaced for being faulty had cracks either in the lateral or longitudinal orientation with their widths 


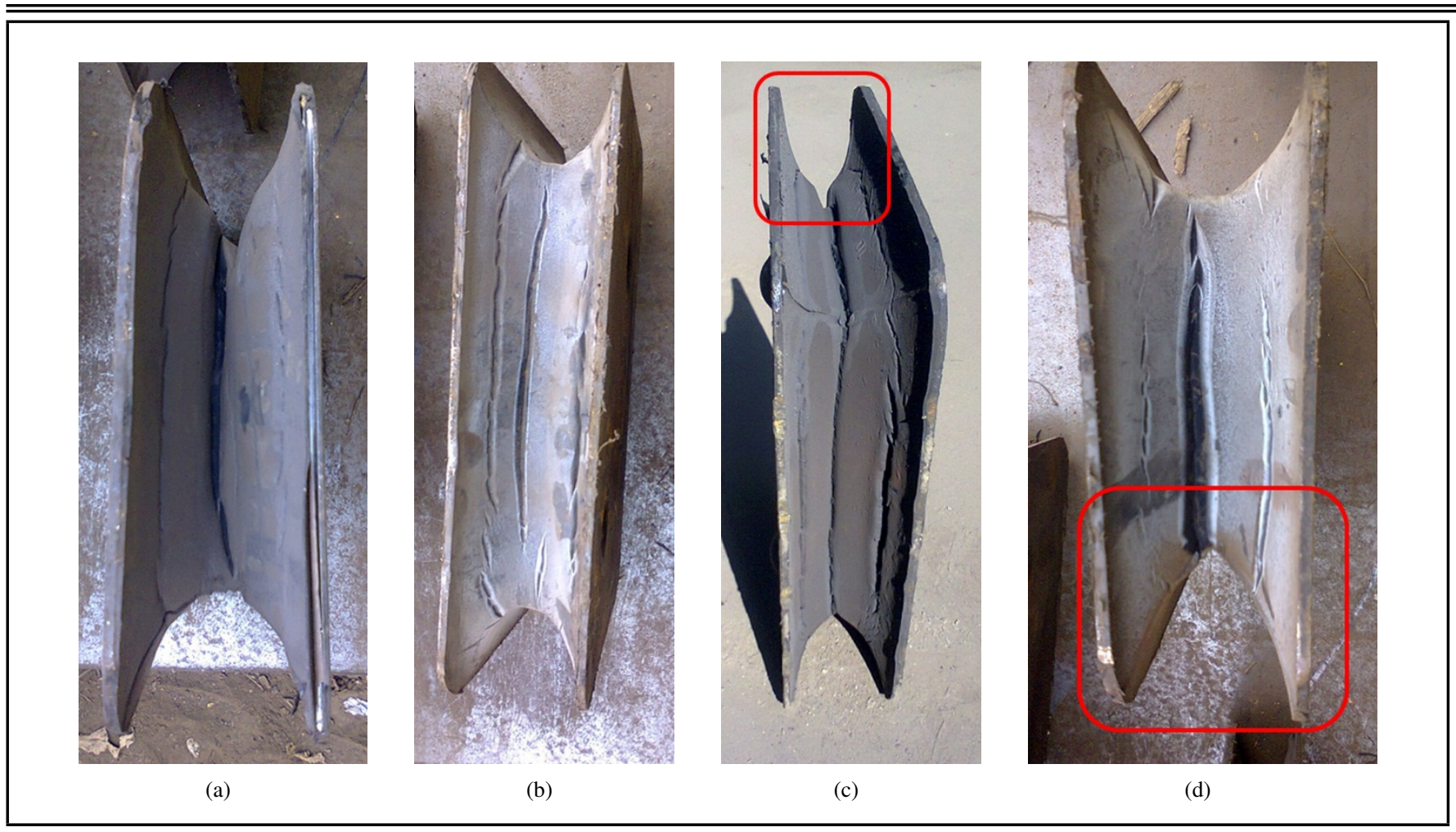

Figure 6. The crack location on the draft pad in (a) the lateral orientation, (b) the longitudinal orientation, (c), and (d) permanent set of rubber compound in the longitudinal direction.

spanning to cover the entire length of the rubber compound in either direction, as seen in Figs. (6a) and (6b). It was also observed that the crack's shape could not be defined by uniform geometry, but its width was spread along the entire length in either the lateral or longitudinal direction of the pad. However, for the simulation, realistic results were achieved by approximating the shape of the crack as semi-elliptical. This semielliptical shape, when defined with an aspect ratio, helped in analysing the dynamics of the draft pad. Furthermore, Figs. (6c) and (6d) describe a permanent set in the rubber compound/draft pad due to the impacting load on the draft gear. This permanent set was observed only on one side of the draft pad, especially the one with a greater length (longitudinal orientation), rather than on the diagonal corners. The effect of such deformation (set), along with fatigue loading, was to promote failure of the rubber compound along the longitudinal direction. Hence, cracks were assumed to be located along the lateral and longitudinal directions rather than at the diagonal corners for current analysis.

The effect of the crack on natural / modal frequency is determined for two different locations on the minimum crosssection of the draft pad, which lies at the geometric centre. Since it is the area and not the volume that affects the natural frequency, the crack's thickness was not treated with importance. Figure 7 illustrates a semi-elliptical crack as part of an ellipse defined by major axis $\mathrm{x}$ and minor axis $\mathrm{y}$. The major axis represents the width of the crack, whereas the semi-minor axis represents the depth of the crack. The aspect ratio for an ellipse is defined as the ratio of its major axis to the minor axis.

Since the crack is semi-elliptical, its aspect ratio was defined by the ratio of the semi-major and the semi-minor axis. Mathematically, the aspect ratio was considered as $x / y$ and was the same as in the case of the crack's lateral and longitudinal orientation. Figures $6 \mathrm{a}$ and $6 \mathrm{~b}$ show the crack spreading along $70-85 \%$ of the entire lateral and longitudinal length at the con-

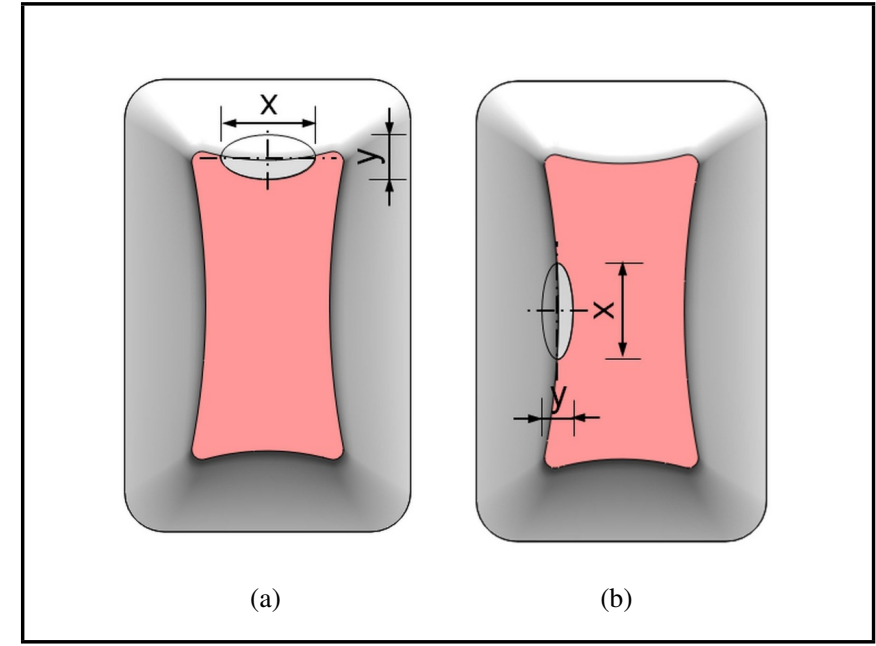

Figure 7. The semi-elliptical crack in the (a) lateral direction and (b) the longitudinal direction.

cerned cross-section. Hence, for the cracks located in these two directions, the width's $(x)$ maximum size was assumed to be $65 \mathrm{~mm}$. This maximum crack size was in proportion to the maximum spread. A lower limit of $40 \mathrm{~mm}$ was considered and the crack size was varied in step of $5 \mathrm{~mm}$. The size $(y)$ of the crack was also varied to achieve aspect the ratios of $0.4,0.5$, $0.6,0.7$, and 0.8 . Table 4 below summarizes the crack sizes for different crack widths and aspect ratios.

\section{RESULTS AND DISCUSSION}

\subsection{Effect of Crack Aspect Ratio on Modal Frequency}

Figures 8 to 12 show the frequency drop for different aspect ratios of a semi-elliptical crack. This variation is plotted as a 


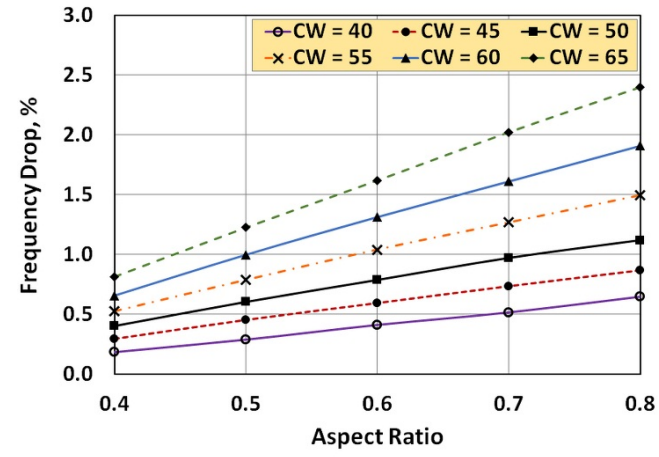

(a)

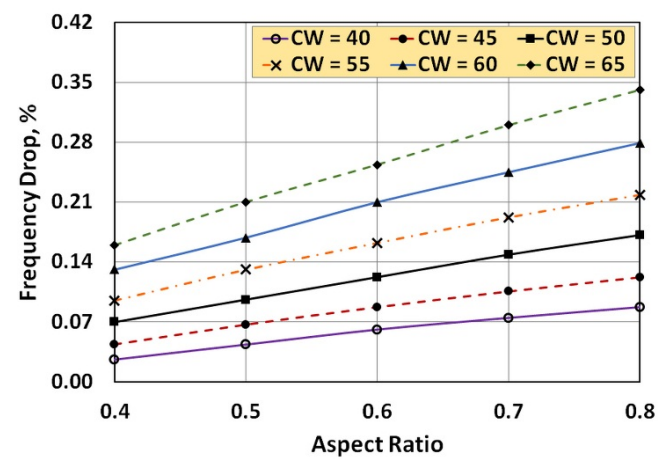

(b)

Figure 8. The effect of the aspect ratio on the first modal frequency for (a) the lateral crack and (b) the longitudinal crack.

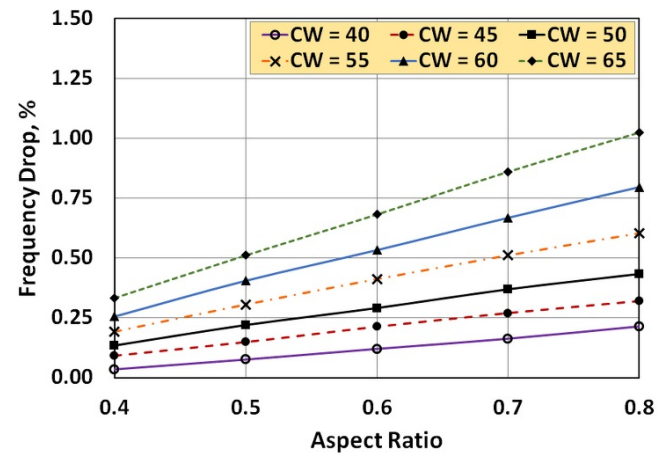

(a)

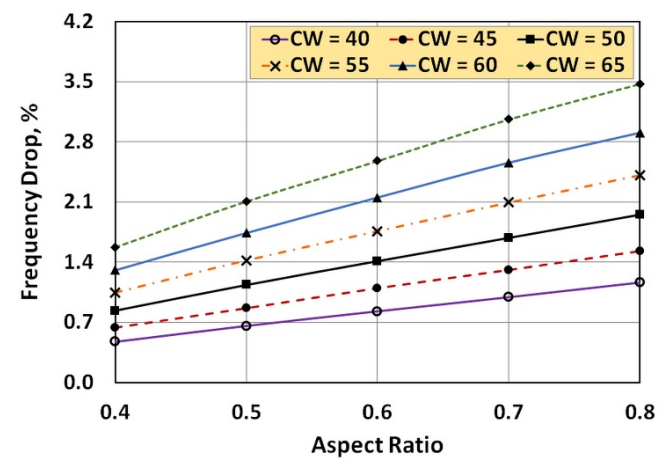

(b)

Figure 9. The effect of the aspect ratio on the second modal frequency for (a) the lateral crack and (b) the longitudinal crack.

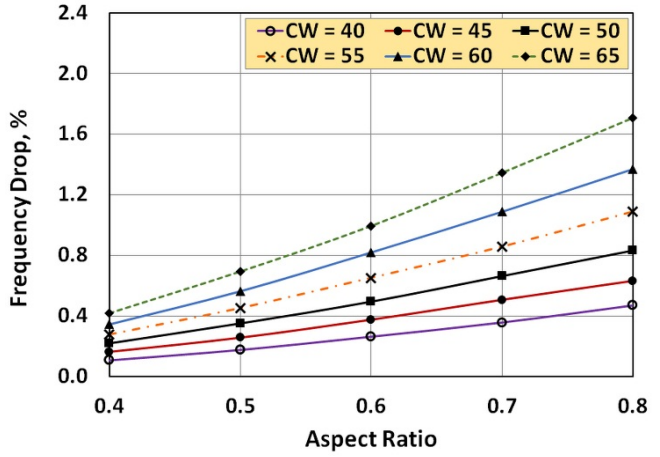

(a)

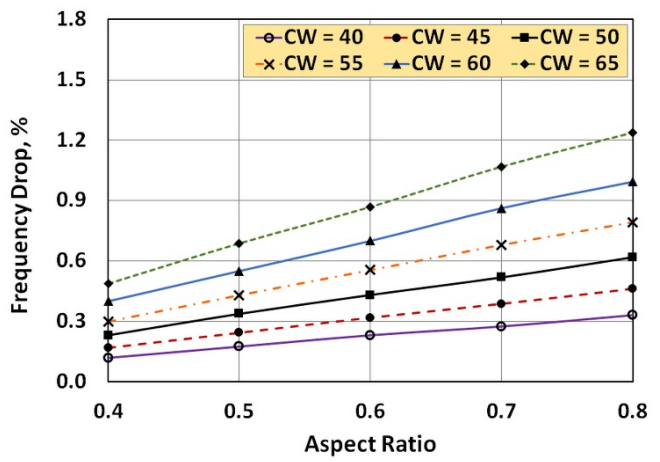

(b)

Figure 10. The effect of the aspect ratio on the third modal frequency for (a) the lateral crack and (b) the longitudinal crack.

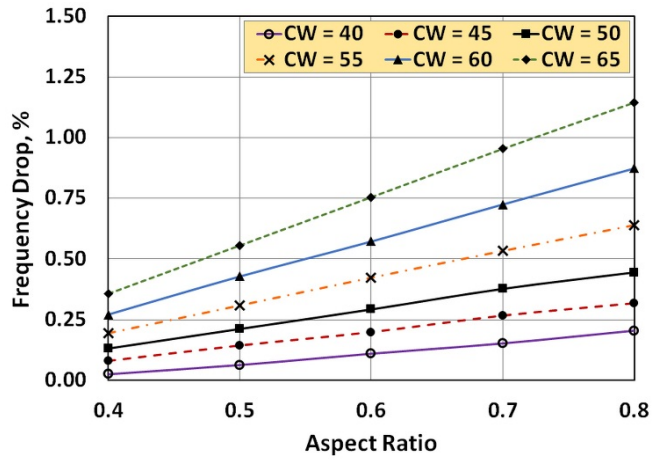

(a)

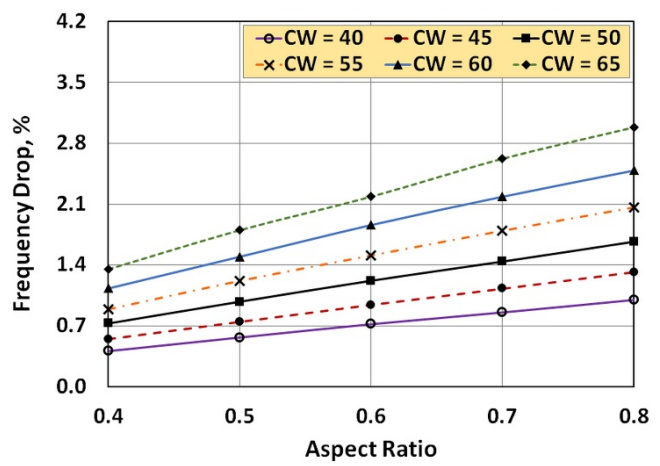

(b)

Figure 11. The effect of the aspect ratio on the fourth modal frequency for (a) the lateral crack and (b) the longitudinal crack. 
Table 4. Depth of the crack for different crack widths and aspect ratios.

\begin{tabular}{|c|c|c|c|c|c||}
\hline Crack width, mm & \multicolumn{5}{|c|}{ Aspect ratio } \\
\hline (major axis 'x') & 0.4 & 0.5 & 0.6 & 0.7 & 0.8 \\
\hline 40 & 8.00 & 10.00 & 12.0 & 14.00 & 16.0 \\
\hline 45 & 9.00 & 11.25 & 13.5 & 15.75 & 18.0 \\
\hline 50 & 10.0 & 12.50 & 15.0 & 17.50 & 20.0 \\
\hline 55 & 11.0 & 13.75 & 16.5 & 19.25 & 22.0 \\
\hline 60 & 12.0 & 15.00 & 18.0 & 21.00 & 24.0 \\
\hline 65 & 13.0 & 16.25 & 19.5 & 22.75 & 27.0 \\
\hline
\end{tabular}

function of the crack width for various modes. The plots reveal that each mode responds differently to the presence of the crack with variation in the aspect ratio. As mentioned in earlier section, the top plate and rubber compound in the first mode shape exhibit an oscillatory motion about the $\mathrm{x}$-axis. This motion is controlled by a component of stiffness in the $\mathrm{z}$ direction. From Figs. $8 \mathrm{a}$ and $8 \mathrm{~b}$, it is evident that as the aspect ratio of the crack increases, the frequency drops. This drop in frequency is almost linear in case of the lateral as well as the longitudinal crack, and the linearity is observed for all the crack widths. It is further observed that the given mode responds differently to the crack's location. For the lateral crack, the drop is significant (maximum 2.40\%), whereas in the longitudinal crack, it is negligible (maximum $0.34 \%$ ), which indicates a sensitivity of the first mode shape to the presence of the lateral crack.

Similarly for the second mode, where the top plate and rubber compound tend to oscillate about y-axis of the base plate of draft pad, the frequency drop is linear, as seen in Figs. 9a and $9 \mathrm{~b}$. The maximum magnitude of the drop is barely $1 \%$ in the case of the lateral crack, whereas for the longitudinal crack for this drop has a significant value (maximum 3.4\%). Thus, the crack's presence in the lateral direction has a minimal effect on the second modal frequency, even for crack widths as high as $65 \mathrm{~mm}$, whereas their presence in the longitudinal orientation causes a drop in frequency of up to $3.5 \%$ for the maximum crack width. For the third mode shape, the top plate and rubber compound oscillate about the z-axis of the base plate. The frequency drop in the presence of a lateral and longitudinal crack is nearly the same and almost linear, as seen in Figs. 10a and $10 \mathrm{~b}$. Moreover, the frequency is not significantly affected. The fourth mode shape shows behaviour similar to that of the second mode. The only difference is that in the second mode, the top plate and rubber compound oscillate about the y-axis of the base plate, whereas for the fourth mode, the top plate and rubber compound oscillate about the y-axis of the top plate. The maximum decrease is $1.14 \%$ for the crack in the lateral direction, whereas the maximum decrease is $3.4 \%$ in the longitudinal direction, as seen in Figs. 11a and 11b.

Fifth mode shape describes the oscillatory motion of the top plate and rubber compound about $\mathrm{z}$-axis of the top plate. This mode shape is most responsive to the presence of lateral crack as compared to all other mode shapes and least responsive to the presence of longitudinal crack as seen from Figs. 12a and $12 \mathrm{~b}$.

\subsection{The Effect of Crack Width on Modal Frequency}

Figures 13 to 17 show the frequency drop for different widths $(x)$ of a semi-elliptical crack. This variation is plotted as a function of the aspect ratio for various modes. It is clear from the plots that for all mode shapes, the frequency drops with an increasing crack width. The increasing aspect ratio further enhances this frequency drop. A significant aspect re-

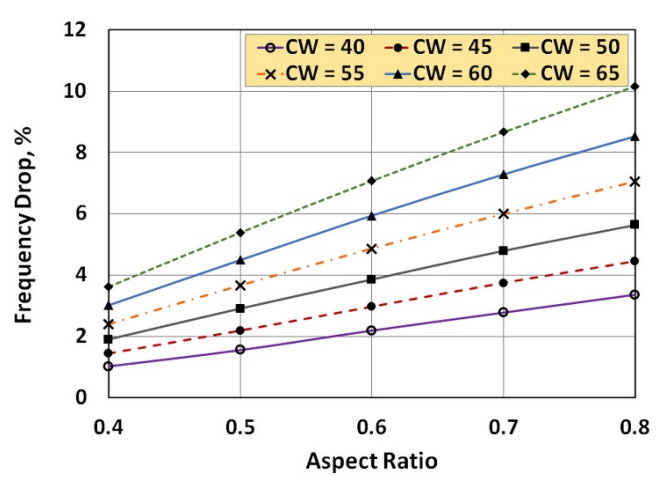

(a)

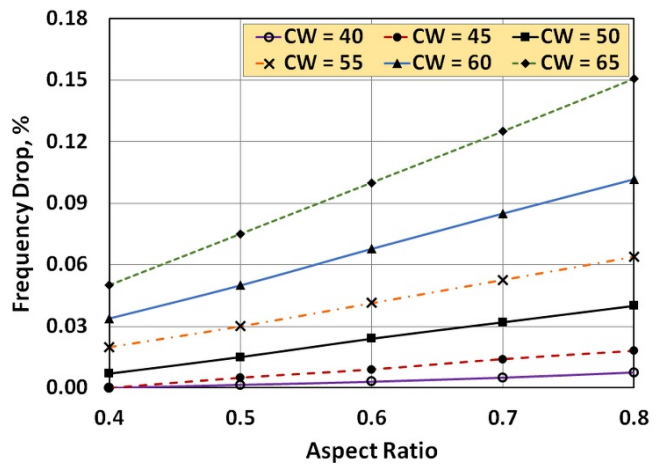

(b)

Figure 12. The effect of the aspect ratio on the fifth modal frequency for (a) the lateral crack and (b) the longitudinal crack.

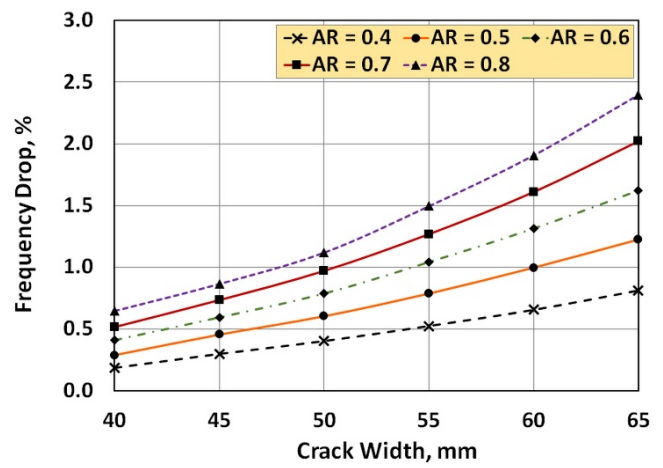

(a)

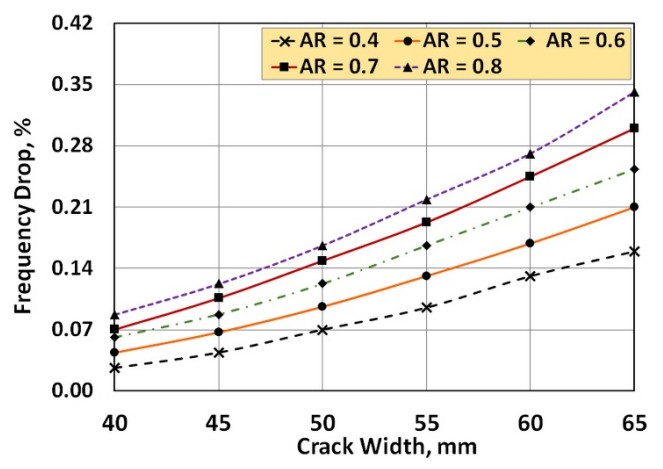

(b)

Figure 13. The effect of the crack width on the first modal frequency for (a) the lateral crack and (b) the longitudinal crack. 


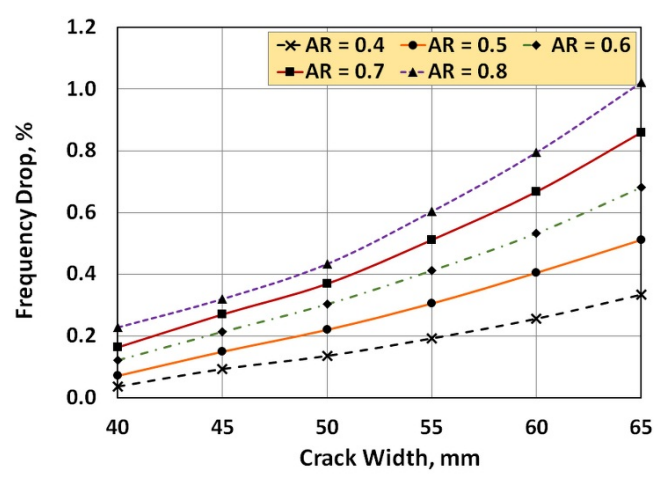

(a)

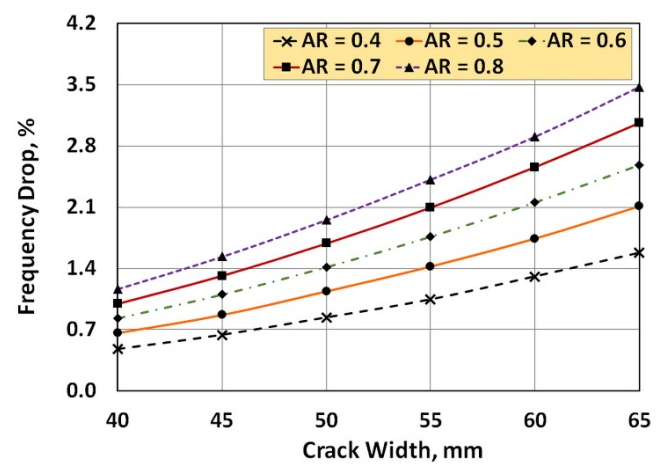

(b)

Figure 14. The effect of the crack width on the first modal frequency for (a) the lateral crack and (b) the longitudinal crack.

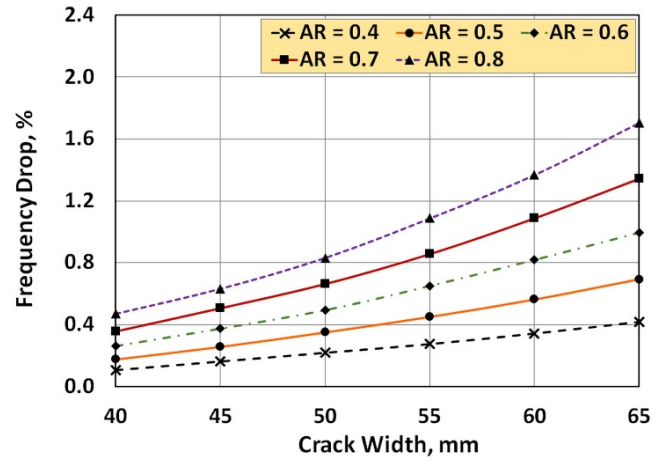

(a)

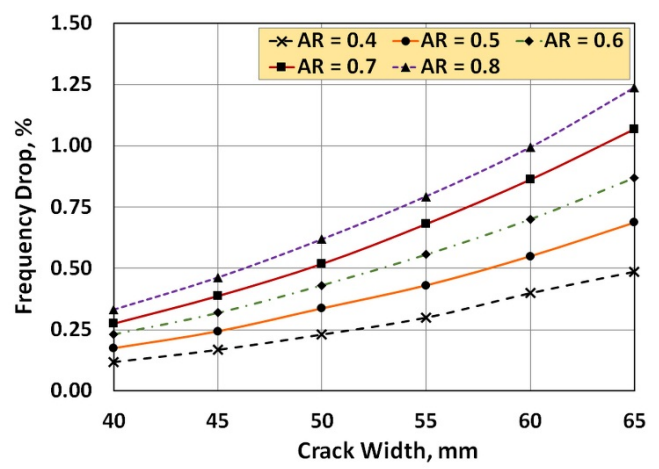

(b)

Figure 15. The effect of the crack width on the first modal frequency for (a) the lateral crack and (b) the longitudinal crack.

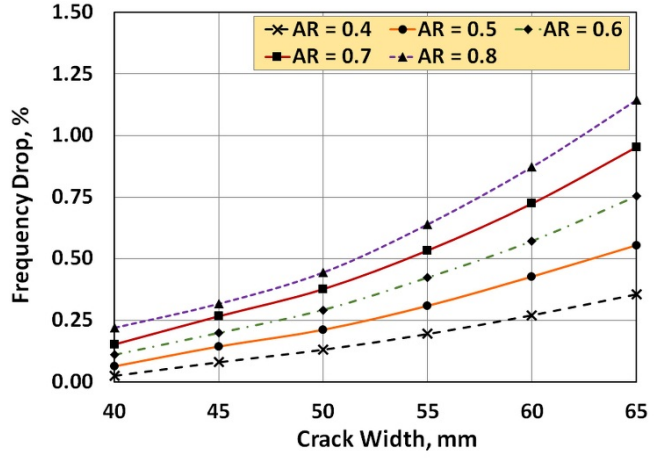

(a)

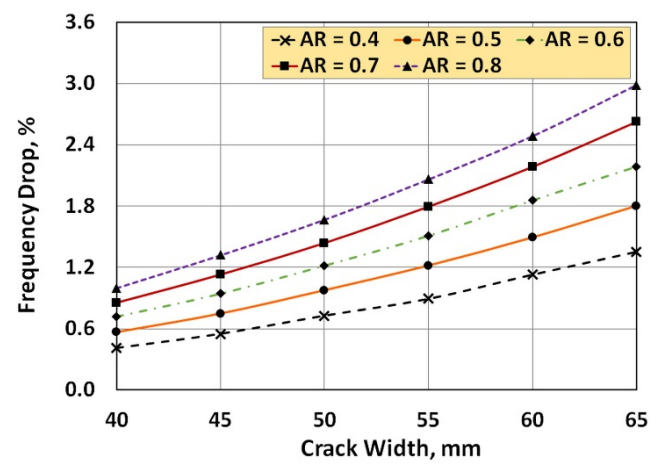

(b)

Figure 16. The effect of the crack width on the first modal frequency for (a) the lateral crack and (b) the longitudinal crack.

garding the frequency drop is that it is parabolic in nature.

Figures $13 \mathrm{a}$ and $13 \mathrm{~b}$ show that the first mode shape is more sensitive to the lateral crack than to the longitudinal crack. Variation in the crack width in the lateral direction causes a significant frequency drop, as compared to the longitudinal direction. The second mode, as seen from Figs. 14a and 14b, is more sensitive to the longitudinal crack width variation as compared to the lateral crack's width variation. Figs. 15a and $15 \mathrm{~b}$ show that the third mode shape responds equally to the lateral as well as the longitudinal crack variation. The fourth mode shape has a response similar to that of the second mode shape for the lateral and longitudinal cracks, as seen from Figs. 16a and 16b. The fifth mode shape is greatly affected by the lateral cracks than the longitudinal cracks. This mode responds very poorly to the longitudinal cracks, as seen in Figs. 17a and 17b.

\section{CONCLUSIONS}

In this work, a modal analysis of draft gear comprising of six compressed draft pads is done with healthy and defective draft pads so that the effect of a defect (i.e. a crack), on the natural frequency of the draft pad can be studied. The mode shapes and frequencies for an individual draft pad and a draft gear are obtained using the finite element approach. Critical observations reveal that crack parameters, such as crack width, crack aspect ratio, and crack location/orientation, influence the dynamic behaviour of the draft pad. The following observations are noteworthy because they reveal the dynamics of the draft pad:

- If the aspect ratio is varied while maintaining the crack 


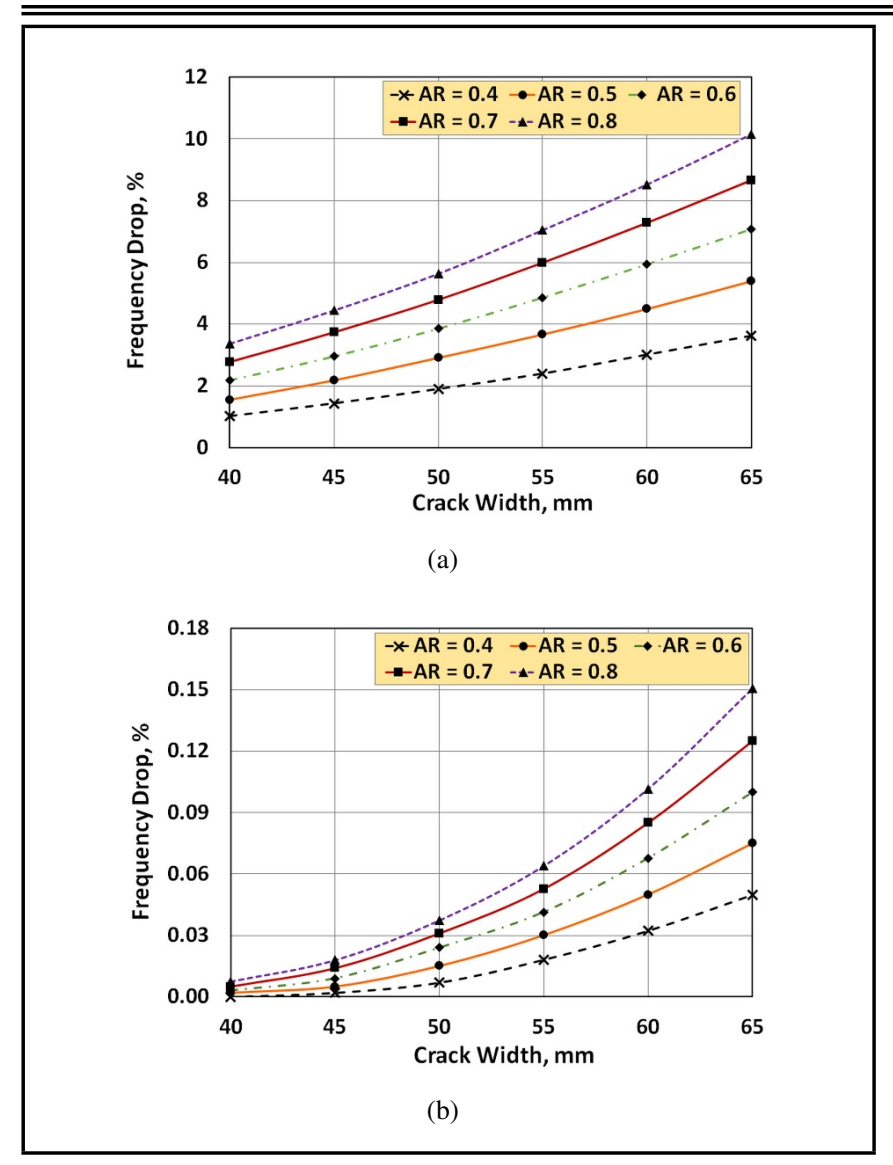

Figure 17. The effect of the crack width on the first modal frequency for (a) the lateral crack and (b) the longitudinal crack.

width constant, then the frequency drop is linear. This linear behaviour can be attributed to the variation of the aspect ratio. Since the crack width is constantly maintained, and the aspect ratio is varied, the reduction in the area becomes a function of depth only.

- If the crack width is varied while constantly maintaining the aspect ratio, then the frequency drop is parabolic. This second order variation can be attributed to the constant value of the aspect ratio, which makes it necessary to change the width while changing the depth.

- It is observed that if the crack lies in the zone of minimum modal displacement, then the frequency drop is minimal. Additionally, if the crack lies in the zone of maximum modal displacement, then the frequency drop is significant.

- It is also observed that frequency reduction is the function of stiffness rather than mass because the presence of the crack affects the area more than the volume. Hence, the presence of the crack is noted through changes in stiffness rather than in mass.

The above observations can be confirmed from Eqs. (4), (5), (6), and (10). Thus, the finite element approach can be used as a reliable tool for predicting dynamic responses of draft pads. This prediction can help to determine the extent to which the frequency values in Table 3, which can vary. If the loading frequency to which the draft gear is subjected due to acceleration and braking of the locomotive are equivalent to the values in Table 3 then failure of draft pads would occur. It is necessary to test the draft pads at these values to determine the fatigue response. Hence, a range of frequency values are identified against which a draft pad must be tested for fatigue response against the cyclic loading of $10 \mathrm{~Hz}{ }^{12}$ This study provides a tool for monitoring exciting frequencies of draft gear and shows how each modal frequency is affected by the crack due to parameters like aspect ratio, crack width, and crack location/orientation.

\section{REFERENCES}

1 Garg, V. K. and Dukkipati, R. V. Dynamics of railway vehicle systems, Academic Press, Canada, (1984). http://dx.doi.org/10.1016/B978-0-12-275950-5.50014-6

2 Duncan, I. B. and Webb, P. A. The longitudinal behaviour of heavy haul trains using remote locomotives, Fourth Int. Heavy Haul Conf., Brisbane, 579-583, 1989.

3 Chen, O. Dynamic simulation of Taipei EMU train, Veh. Syst. Dyn.: Int. J. Veh, Mech. Mob., 30 (2), 143-167, (1998). http://dx.doi.org/10.1080/00423119808969441

4 McClanachan, M., Cole, C., Roach, D. and Scown B. An investigation of the effect of bogie and wagon pitch associated with longitudinal train dynamics, Veh. Syst. Dyn.: Int. J. Veh, Mech. Mob., 33 (Supplement), 374-385, (1999).

5 Cole, C. and Sun, Y. Q. Simulated comparisons of wagon coupler systems in heavy haul trains, Proc. Inst. Mech. Eng. F J. Rail Rapid Transit, 220 (3), 247-256, (2006). http://dx.doi.org/10.1243/09544097JRRT35

6 Belforte, P., Cheli, F., Diana, G., and Melzi, S. Numerical and experimental approach for the evaluation of severe longitudinal dynamics of heavy freight trains, Veh. Syst. Dyn.: Int. J. Veh, Mech. Mob., 46 (S1), 937-955, (2008). http://dx.doi.org/10.1080/00423110802037180

7 Nasr, A. and Mohammadi, S. The effects of train delay time on in-train forces, Proc. Inst. Mech. Eng. F J. Rail Rapid Transit, 224 (3), 523-534, (2010). http://dx.doi.org/10.1243/09544097JRRT306

8 Cole, C., McClanachan, M., Spiryagin, M., and Sun, Y. Q. Wagon instability in long trains, Veh. Syst. Dyn.: Int. J. Veh, Mech. Mob., 50 (Supplement), 303-317, (2012). http://dx.doi.org/10.1080/00423114.2012.659742

9 Qi, Z., Huang, Z., and Kong, X. Simulation of longitudinal dynamics of long freight trains in positioning operations, Veh. Syst. Dyn.: Int. J. Veh, Mech. Mob., 50 (9), 1409-1433, (2012). http://dx.doi.org/10.1080/00423114.2012.661063

10 Wu, Q., Luo, S., and Cole, C. Longitudinal dynamics and energy analysis for heavy haul trains, J. Mod. Transport., 22 (3), 127-136, (2014). http://dx.doi.org/10.1007/s40534014-0055-X

11 Rao, S. S. Mechanical Vibrations, Pearson Education, New Delhi, India, (2009).

12 Research Design \& Standards Organisation, Ministry of Railways, Lucknow - 226011. Amendment No. 3 of September, 1993 to Indian Railways Schedule Of Technical Requirement No. 55-Bd-90 For Rubber Springs of High Capacity Draft Gear For Centre Buffer Couplers of Bg Bogie Wagons Having 625.5 mm Draft Gear Pocket, (1993). 